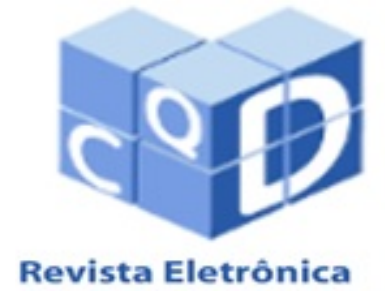

Paulista de Matemática

ISSN 2316-9664

Volume 10, dez. 2017

Edição Ermac

\section{Luca Meacci \\ Instituto de Ciências \\ Matemáticas e de Computação \\ Universidade de São Paulo \\ luca.meacci@usp.br}

Franciane Fracalossi Rocha

Instituto de Ciências

Matemáticas e de Computação

Universidade de São Paulo

fr.franciane@usp.br

\section{Arianne Alves Silva \\ Instituto de Ciências \\ Matemáticas e de Computação \\ Universidade de São Paulo \\ arianne@usp.br}

\section{Petterson Vinicius Pramiu}

Instituto de Ciências

Matemáticas e de Computação

Universidade de São Paulo

ppramiu@usp.br

\title{
Planejamento de trajetória para a manipulação de partículas em microfluídica
}

Trajectory planning for microfluidic particle manipulation

\begin{abstract}
Resumo
Partículas imersas em fluidos são transportadas através de campos de velocidade. É de interesse de problemas com importantes aplicações em microfluídica ter-se um controle sobre como as partículas imersas transportam-se no fluido. Uma forma de controlar-se a trajetória das partículas é o uso de atuadores de sistema para induzir campos de velocidades adequados ao trajeto desejado. Nesse trabalho propõe-se uma primeira abordagem como ferramenta básica para o estudo de planejamento de trajetória ótima para o transporte de uma partícula de um ponto a outro em um domínio com presença de obstáculos e atuadores do tipo imposição de pressão ou imposição de voltagem através de eletrodos.
\end{abstract}

Palavras-chave: Mecânica dos Fluidos e Aplicações. Planejamento ótimo de trajetória. Manipulação de partículas. Princípio de Bellman. Equação de Hamilton-Jacobi.

Abstract
Particles immersed in fluids are transported through velocity fi-
elds. It is of interest to problems with important applications in
microfluidics to have a control over how the immersed particles
are transported in the fluid. One way to control the trajectory of
the particles is to use system actuators to induce fields of velo-
cities appropriate to the desired path. In this work we propose a
first approach as basic tool for studying the optimum trajectory
planning for the transport of a particle from one point to another
in a domain with presence of obstacles and actuators of the type
imposition of pressure or imposition of voltage through electro-
des.
Keywords: Mechanics of Fluids and Applications. Planning of
the trajectory. Manipulation of particles. Principle of Bellman.
Hamilton-Jacobi equation.

Gustavo Carlos Buscaglia

Instituto de Ciências

Matemáticas e de Computação

Universidade de São Paulo

gustavo.buscaglia@icmc.usp.br 


\section{Introdução}

Manipular o transporte, de um ponto a outro, de partículas imersas em um fluido é um problema de grande interesse atual e importante no campo de aplicações. Vários estudos têm sido dirigido nessa direção, tais como nos artigos de Armani et al. (2005) e Tuval et al. (2005). O trabalho de Satej Chaudhary e Benjamin Shapiro (2006) apresenta um estudo de governo de partículas múltiplas em um sistema microfluídico eletrosmoticamente guiado. Neste trabalho mencionado, é feita referência à possibilidade de considerar um problema de planejamento de caminho, ou seja, procurar as melhores trajetórias para transportar partículas de locais iniciais a finais específicos. No entanto, esta questão é deixada em aberto e não discutida. Em nosso trabalho aqui apresentado, considera-se como configuração física o espaço limitado entre duas paredes paralelas, onde um fluxo é induzido por atuadores de maneira controlada. Os atuadores considerados são pressão ou voltagem, que são ativados de maneira a conduzir o fluxo e, consequentemente, as partículas que fluem imersas no fluido. O estudo é dirigido de maneira de propor uma primeira abordagem que representa uma base para determinar-se a trajetória ótima, em termos de tempo, para as partículas que serão transportadas a partir de um ponto de partida para um ponto de chegada. Para atingir este objetivo, será formalizado matematicamente o problema na seção 2, amplamente descrito e resolvido numericamente o cálculo do campo de velocidade induzido por todos os controles admissíveis na seção 3 e, finalmente, na seção 4 são mostrados os primeiros resultados das simulações para o problema de planejamento de trajetória ótima em termos de tempo mínimo de acordo com o princípio da Bellman, a partir do qual é derivada a equação de Hamilton-Jacobi.

Este artigo expande o trabalho apresentado oralmente no IV ERMAC - Encontro Regional de Matemática Aplicada e Computacional na Unesp em Bauru SP (Brasil) e publicado em forma resumida no Caderno de trabalhos completos e resumos do IV ERMAC (MEACCI et al., 2017).

\section{O problema}

O domínio considerado é o quadrado $(0, L)^{2}=\Omega$, para o escoamento de um fluido sem inércia com a presença de obstáculos e também de atuadores $X^{N}, X^{S}, X^{E}$ e $X^{W}$ em cada lado do domínio, que podem ser pressão ou tensão. Na Figura 1 tem-se uma exemplificação para o tipo de configuração de domínio que será considerada. Percebe-se que para levar uma partícula de um ponto inicial a um ponto final será determinada uma trajetória que é influenciada pela ação dos atuadores e pela posição dos obstáculos.

Os valores $\underline{X}$ impostos nos atuadores pertencem a um conjunto admissível de controles $\mathscr{A}$ e geram um campo de velocidades $\mathbf{v}(\vec{x}, \underline{X})$ em cada ponto (CHAUDHARY e SHAPIRO, 2006). Seja $P$ uma partícula cuja posição inicial é $\vec{x}^{0}$. Cada função de controle $\underline{X}(\cdot):[0, T] \rightarrow \mathscr{A}$ movimenta $P$ ao longo de uma trajetória $\vec{r}(t)$ que é a solução de

$$
\left\{\begin{array}{l}
\frac{d \vec{r}}{d t}=\mathbf{v}(\vec{r}(t), \underline{X}(t)) \\
\vec{r}(0)=\vec{x}_{0} .
\end{array}\right.
$$

O objetivo deste trabalho é determinar uma trajetória $\vec{r}(t)$ tal que para $t^{*}<T$ seja $\vec{r}\left(t^{*}\right) \in Z$, sendo $Z$ o conjunto alvo. A trajetória útil nas aplicações não deve colidir com paredes nem com obstáculos, e deve evitar regiões do domínio onde os controladores não conseguem movimentar 


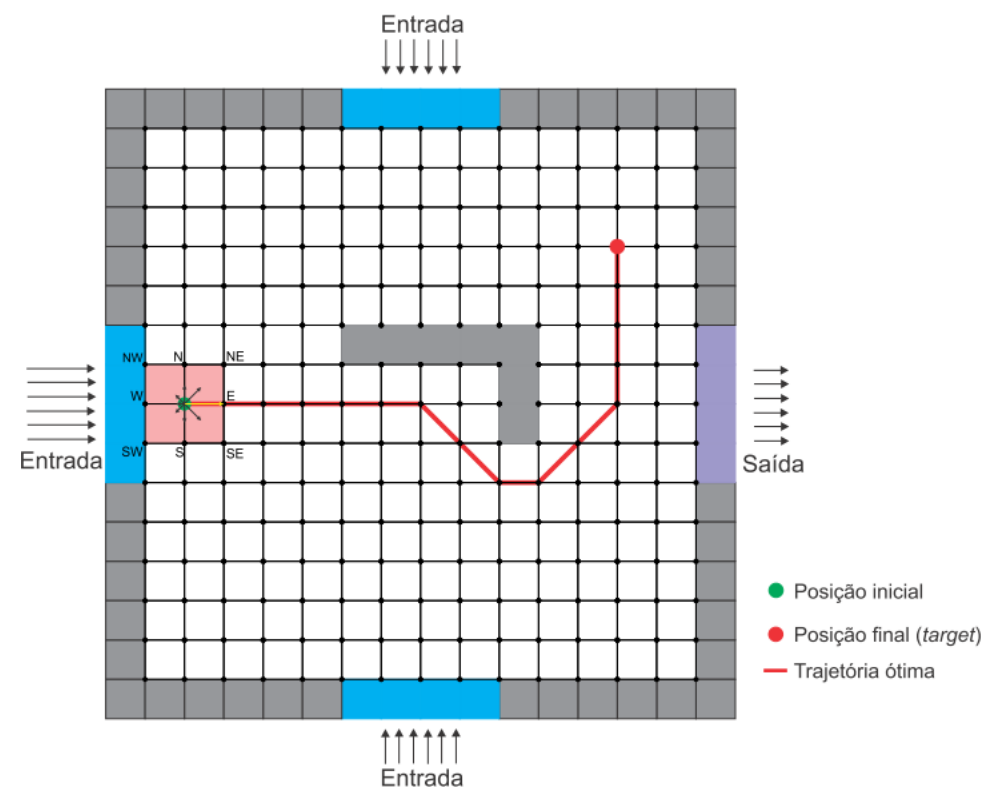

Figura 1: Esquema do problema.

significativamente o fluido (regiões "mortas"). Por isso, como primeiro enfoque ao problema, foi escolhida, como trajetória da partícula sendo manipulada, aquela que minimiza o tempo em atingir o alvo.

Para formalizar matematicamente o problema começamos definindo a velocidade máxima possível de ser gerada na direção $\breve{d}$ como a solução do seguinte problema de otimização:

$$
V(\vec{x}, \breve{d})=\max _{\underline{X} \in \mathscr{A}} \mathbf{v}(\vec{x}, \underline{X}) \cdot \check{d} .
$$

Para um ponto $\vec{x} \in \Omega$ podemos considerar a função $T(\vec{x})$ definida como o mínimo tempo necessário para levar $P$ de $\vec{x} a Z$. Associada a $T(\vec{x})$ existe uma trajetória ótima, que leva $P$ a $Z$ no tempo mínimo.

O princípio de Bellman estabelece que, se um ponto $\vec{y}$ está na trajetória ótima $\Gamma$ de $\vec{x}$, então a trajetória ótima de $\vec{x}$ a $\vec{y}$ é o arco de $\Gamma$ que une esses pontos, e a trajetória ótima de $\vec{y}$ ao alvo $Z$ é o arco final de $\Gamma$. Com esse princípio é possível chegar à equação de Hamilton-Jacobi-Bellman para $T$ :

$$
V(\vec{x}, \check{v}(\vec{x})) \check{v}(\vec{x}) \cdot \nabla T+1=0,
$$

onde $\check{v}(\vec{x})$ é dado por $\check{v}(\vec{x})=\arg \min _{\|\check{d}\|=1} V(\vec{x}, \breve{d}) \check{d} \cdot \nabla T(\vec{x})$ e $\check{d}$ é a direção do deslocamento.

A propriedade fundamental que usaremos no tratamento numérico dessa equação (veja em (CRISTIANI, 2013)) é a seguinte:

Proposição Seja $\vec{x} \in \Omega$, e seja $\gamma$ uma curva (superfície em $3 D$ ) fechada tal que $\vec{x} e ́$ interior a $\gamma$, e o alvo $Z$ é exterior a $\gamma$. Para cada $\vec{y} \in \gamma$, seja $\zeta(\vec{y})$ o tempo mínimo requerido para levar uma partícula de $\vec{x}$ a $\vec{y}$. Então, $T(\vec{x})=\min _{\vec{y} \in \gamma}\{T(\vec{y})+\zeta(\vec{y})\}$.

Se a curva $\gamma$ é suficientemente pequena podemos considerar as trajetórias retas entre $\vec{x}$ e $\gamma$, donde 


$$
T(\vec{x})=\min _{\vec{y} \in \gamma}\left\{T(\vec{y})+\frac{\|\vec{y}-\vec{x}\|}{V\left(\vec{x}, \frac{\vec{y}-\vec{x}}{\|\vec{y}-\vec{x}\|}\right)}\right\}
$$

e a trajetória ótima, localmente, é o segmento $\overline{\vec{x}} \overrightarrow{\text {. }}$.

Com a trajetória ótima determinada localmente é possível determinar-se a trajetória ótima global para qualquer ponto do domínio ser deslocado até o ponto final, como uma união das trajetórias ótimas locais, de acordo com o princípio de Bellman. O problema numérico que consideramos, portanto, é o de discretizar e resolver a equação (4), para o qual devemos construir primeiramente o campo $V(\vec{x}, \breve{d})$.

\section{Cálculo de $V(\vec{x}, \breve{d})$}

Para calcular o conjunto $V(\vec{x}, \breve{d})$ avaliamos os campos de velocidades $\mathbf{v}(\vec{x}, \underline{X})$ em cada ponto, que dependem das condições de contorno impostas nos atuadores. Essa dependência é linear, de modo que os campos de velocidades podem ser avaliados diretamente como combinação linear da solução sobre uma base, como mostrado nas próximas subseções.

Considerando-se o caso em que o campo de velocidades é gerado por fontes de pressão, temse que a equação governante para o fluxo fluídico é a equação de Stokes

$$
-\mu \nabla^{2} \mathbf{v}+\nabla p=0, \quad \nabla . \mathbf{v}=0 \mathrm{em} \Omega .
$$

com condições de contorno de Dirichlet nos atuadores (pressão) e obstáculos e paredes (velocidade nula).

Para os atuadores do tipo eletrodos gera-se um campo elétrico, que é governado pela equação de Poisson homogênea para o potencial elétrico $\nabla^{2} \Phi=0$, que relaciona-se com o campo elétrico $\mathbf{E}=-\nabla \Phi$. Por sua vez, o campo elétrico induz um campo de velocidade de acordo com a relação $\mathbf{v}=m_{e o} \mathbf{E}$, onde $m_{e o}$ é a constante de eletrosmótica relativa à interação entre o fluido e a parede. As condições de contorno são de Dirichlet para os atuadores (voltagem imposta) e de Neumann homogêneas para as paredes e obstáculos (BUSCAGLIA, 2016).

\subsection{Atuadores do tipo pressão: equação de Stokes}

Considerando-se o caso em que o campo de velocidades é gerado por fontes de pressão, tem-se que a equação governante para o fluxo fluídico é a equação de Stokes (5). O termo $\nabla . v=0$ garante a conservação de massa para um fluido newtoniano incompressível. Já o termo $-\mu \nabla^{2} \mathbf{v}+\nabla p=$ 0 garante a conservação do momento no caso em que o termo de inércia não é considerado. Considera-se também que termo fonte é nulo e o caso estacionário, de acordo com a equação.

A equação de Stokes será resolvida numericamente utilizando-se o método de discretização MAC (Marker and Cell), considerando-se o esquema ilustrado na Figura 2 e respectiva notação. Seguem listadas algumas especificações:

- O domínio $\Omega$ está discretizado com $\left(N_{x}=N 1+2\right) \times\left(N_{y}=N 2+2\right)$ células de pressão, cujos extremos são $X_{i}, X_{i+1}, Y_{j}$ e $Y_{j+1}$. A incógnita de pressão $P_{i, j}$ é situada no centro da célula, nas coordenadas $\left(\hat{X}_{i}, \hat{Y}_{j}\right)$. A primeira e última linhas e colunas são células dummy 


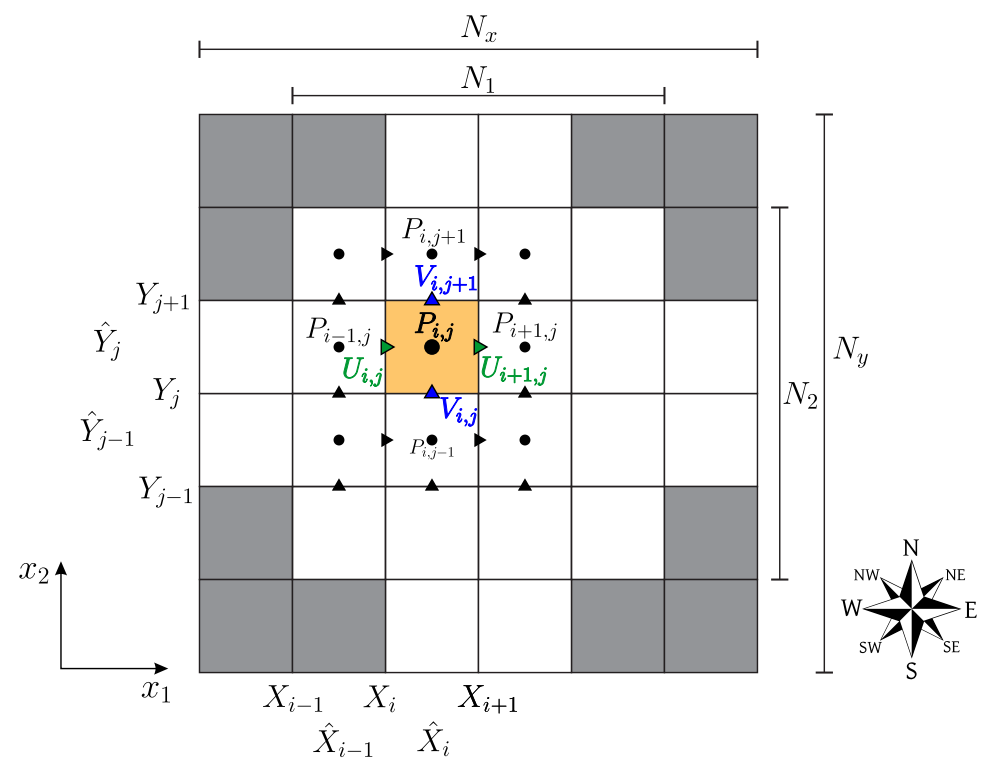

Figura 2: Ilustração do posicionamento das variáveis pressão e velocidade.

modelo que se encontram fora de $\Omega$ e são utilizadas somente para especificar as condições de contorno.

- As variáveis de velocidade na direção $x_{1}, U_{i, j}$ e $U_{i+1, j}$ são situadas nas coordenadas $\left(X_{i}, \hat{Y}_{j}\right)$ e $\left(X_{i+1}, \hat{Y}_{j}\right)$ e aparecem nos centros dos lados leste e oeste da célula de pressão. O número total dessas variáveis é $\operatorname{dim}(U)=\left(\left(N_{x}+1\right) N_{y}\right)$.

- As variáveis de velocidade na direção $x_{2}, V_{i, j}$ e $V_{i, j+1}$ são situadas nas coordenadas $\left(\hat{X}_{i}, Y_{j}\right)$ e $\left(\hat{X}_{i}, Y_{j+1}\right)$ e aparecem nos centros dos lados norte e sul da célula de pressão. O número total dessas variáveis é $\operatorname{dim}(V)=\left(\left(N_{y}+1\right) N_{x}\right)$.

- A distância entre $X_{i}$ e $X_{i+1}$ é denotada por $h_{x}$. A distância entre $Y_{j}$ e $Y_{j+1}$ denota-se por $h_{y}$. Por sua vez, $\hat{h}_{x}$ e $\hat{h}_{y}$ denotam as distâncias entre $\hat{X}_{i}$ e $\hat{X}_{i+1}$ e $\hat{Y}_{j}$ e $\hat{Y}_{j+1}$, respectivamente.

Com essa configuração, a conservação da massa é dada por

$$
\left(u_{E}^{*}-u_{W}^{*}\right) h_{y}+\left(v_{N}^{*}-v_{S}^{*}\right) h_{x}=0,
$$

onde $v_{N}^{*}, v_{S}^{*}, u_{E}^{*}$ e $u_{W}^{*}$ são as velocidades médias sobre as faces norte, sul, leste e oeste, respectivamente. A equação (6) conduz a equação de conservação de massa em relação ao volume finito $(i, j)$, da forma

$$
\left(U_{i+1, j}-U_{i, j}\right)\left(Y_{j+1}-Y_{j}\right)+\left(V_{i+1, j}-V_{i, j}\right)\left(X_{i+1}-X_{i}\right)=0 .
$$

Para a conservação do momento na direção $x_{1}$, cujo volume finito está ilustrado na Figura 3, tem-se

$$
-\left(\int_{\partial V}\left(p \check{\mathbf{n}}-2 \mu\left(\nabla \mathbf{u}+\nabla \mathbf{u}^{\mathbf{T}}\right) \check{\mathbf{n}}\right)_{x} d S=0\right.
$$

O termo de pressão é dado por 


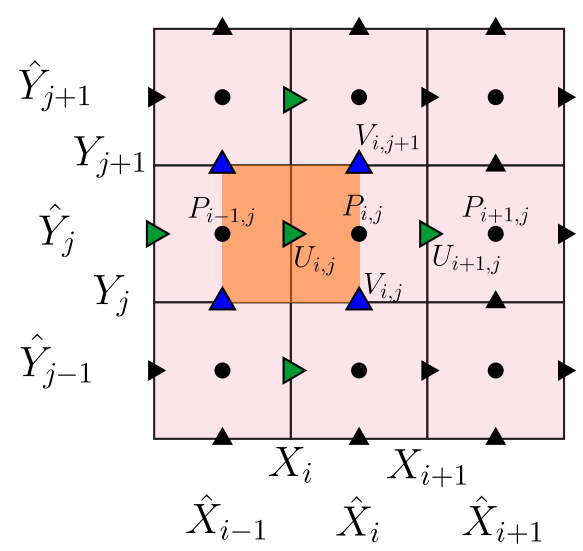

Figura 3: Ilustração do posicionamento da variável $U_{i, j}$.

$$
\int_{\partial V} p n_{x} d S \simeq\left(p_{E}-p_{W}\right) h_{y}=\left(P_{i, j}-P_{i-1, j}\right)\left(Y_{j+1}-Y_{j}\right)
$$

Os termos viscosos em cada face são dados por

- Face norte:

$$
-\int_{N} \mu\left(\partial_{y} u+\partial_{x} v\right) d x=-\mu\left(\frac{U_{i, j+1}-U_{i, j}}{\hat{Y}_{j+1}-\hat{Y}_{j}}+\frac{V_{i, j+1}-V_{i-1, j+1}}{\hat{X}_{i}-\hat{X}_{i-1}}\right)\left(\hat{X}_{i}-\hat{X}_{i-1}\right)
$$

- Face sul:

$$
+\int_{S} \mu\left(\partial_{y} u+\partial_{x} v\right) d x=\mu\left(\frac{U_{i, j}-U_{i, j-1}}{\hat{Y}_{j}-\hat{Y}_{j-1}}+\frac{V_{i, j}-V_{i-1, j}}{\hat{X}_{i}-\hat{X}_{i-1}}\right)\left(\hat{X}_{i}-\hat{X}_{i-1}\right)
$$

- Face leste:

$$
-\int_{E} 2 \mu \partial_{x} u d y=-2 \mu \frac{U_{i+1, j}-U_{i, j}}{X_{i+1}-X_{i}}\left(Y_{j+1}-Y_{j}\right)
$$

- Face oeste:

$$
+\int_{W} 2 \mu \partial_{x} u d y=2 \mu \frac{U_{i, j}-U_{i-1, j}}{X_{i}-X_{i-1}}\left(Y_{j+1}-Y_{j}\right)
$$

Analogamente, é possível determinar os termos para a conservação do momento na direção $x_{2}$. Nota-se que o volume finito neste caso não é o mesmo para quando consideramos as equações na direção $x_{1}$, como é ilustrado na Figura 4.

As incógnitas que se encontram no contorno do domínio, são variáveis "dummy" e devem receber um valor arbitrário de acordo com a condição de Dirichlet. Os obstáculos devem receber o mesmo tratamento que as paredes. Para o caso considerado tem-se a condição de velocidade nula nas paredes e obstáculos. Tem-se a seguir um exemplo do tratamento aplicado à condição de contorno para uma configuração envolvendo células fluídicas e células de parede ou obstáculo.

Considera-se a configuração ilustrada na Figura 5, onde tem-se duas células fluídicas $(i, j) \mathrm{e}$ $(i-1, j)$. Toma-se, por exemplo, o balanço do fluxo para o termo viscoso da célula, que tem como variável de velocidade $U_{i, j}$. Para determinar o fluxo na face norte deve-se calcular a respectiva contribuição $-\int_{N} \mu\left(\partial_{y} u+\partial_{x} v\right) d x$. Tal contribuição depende da natureza das células vizinhas. 


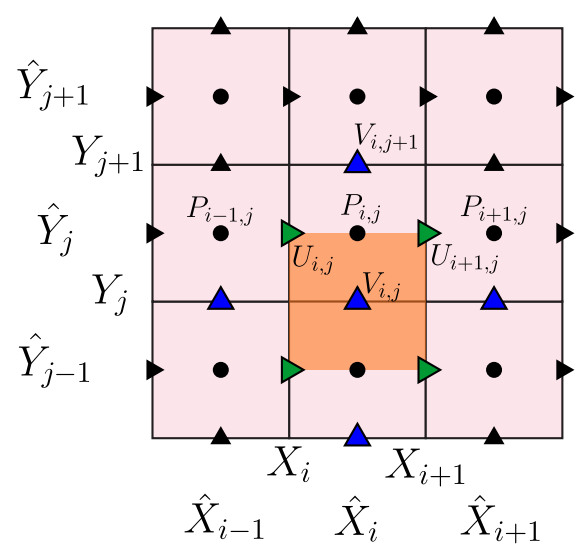

Figura 4: Ilustração do posicionamento da variável $V_{i, j}$.

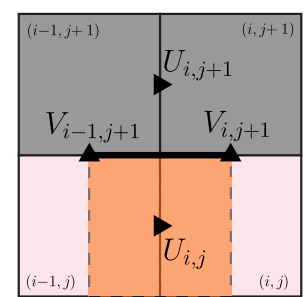

(a) Caso 1.

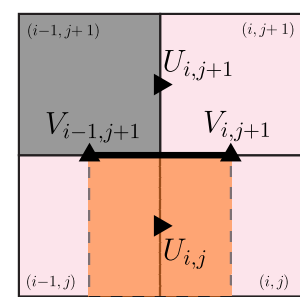

(b) Caso 2.

Figura 5: Configuração para diferentes arranjos entre célula fluida e obstáculo ou parede.

Alguns casos possíveis, com presença de parede ou obstáculo, para as células vizinhas ao norte são:

- Caso 1: as células $(i-1, j+1)$ e $(i, j+1)$ são ambas parede ou obstáculo, onde tem-se:

$$
-\int_{N} \mu\left(\partial_{y} u+\partial_{x} v\right) d x=-\mu\left(\frac{U_{i, j+1}-U_{i, j}}{\left(\hat{Y}_{j+1}-\hat{Y}_{j}\right) / 2}+\frac{V_{i, j+1}-V_{i-1, j+1}}{\hat{X}_{i}-\hat{X}_{i-1}}\right)\left(\hat{X}_{i}-\hat{X}_{i-1}\right),
$$

diferentemente da equação (10), a componente relativa a $\partial_{y} u$ é avaliada em metade da distância pois considera-se o valor para a variável $U_{i, j+1}$ assumido no começo da parede ou obstáculo.

- Caso 2: a célula $(i-1, j+1)$ é parede ou obstáculo e a célula $(i, j+1)$ é fluido, tem-se:

$$
-\int_{N} \mu\left(\partial_{y} u+\partial_{x} v\right) d x=-\mu\left(\frac{U_{i, j+1}-U_{i, j}}{\left(\hat{Y}_{j+1}-\hat{Y}_{j}\right) / 2}+\frac{V_{i, j+1}-V_{i-1, j+1}}{\left(\hat{X}_{i}-\hat{X}_{i-1}\right) / 2}\right)\left(\hat{X}_{i}-\hat{X}_{i-1}\right),
$$

nesse casos as duas componentes relativas a $\partial_{y} u$ e $\partial_{x} v$ são avaliada em metade da distância pois considera-se, novamente, os valores para as variáveis $U_{i, j+1}$ e $V_{i-1, j+1}$ assumidos no começo da parede ou obstáculo.

Outras configurações entre as células podem ser tratadas de modo similar. 


\subsection{Atuadores do tipo eletrodos: campo elétrico}

Para atuadores do tipo eletrodos, ou seja, quando impõe-se uma voltagem, gera-se um campo elétrico, que é governado pela equação de Poisson homogênea para o potencial elétrico $\Phi$

$$
\nabla^{2} \Phi=0
$$

onde impôs-se condição de Dirichlet para os elétrodos e condição de Neumann nula nas parede e obstáculos. O potencial elétrico relaciona-se com o campo elétrico da seguinte maneira

$$
E=-\nabla \Phi
$$

Por sua vez, o campo elétrico induz um campo de velocidade de acordo com a relação

$$
\mathbf{v}=m_{e o} \mathbf{E}
$$

onde $m_{e o}$ é a constante de eletrosmótica relativa à interação entre o fluido e a parede.

A discretização MAC que será utilizada coincide com a discretização na figura 2, sendo que as variáveis de potencial elétrico estão na mesma posição que as variáveis de pressão e as posições das componentes de velocidade são mantidas. Considerando-se um volume de controle, aplica-se o balanço de fluxos para a equação (14). Para a face norte tem-se

$$
\int_{N} \partial_{y} \phi d S=\frac{\phi_{i, j+1}-\phi_{i, j}}{\hat{Y}_{j+1}-\hat{Y}_{j}}\left(X_{i+1}-X_{i}\right) .
$$

Para a face sul a discretização do potencial é

$$
\int_{S} \partial_{y} \phi d S=\frac{\phi_{i, j}-\phi_{i, j-1}}{\hat{Y}_{j}-\hat{Y}_{j-1}}\left(X_{i+1}-X_{i}\right) .
$$

Para a face leste

$$
\int_{E} \partial_{x} \phi d S=\frac{\phi_{i+1, j}-\phi_{i, j}}{\hat{X}_{i+1}-\hat{X}_{i}}\left(Y_{j+1}-Y_{j}\right)
$$

Finalmente, para a face oeste tem-se

$$
\int_{W} \partial_{x} \phi d S=\frac{\phi_{i, j}-\phi_{i-1, j}}{\hat{X}_{i}-\hat{X}_{i-1}}\left(Y_{j+1}-Y_{j}\right)
$$

As condições de contorno consideradas levam em conta o caso onde tem-se eletrodo e o caso onde tem-se parede ou obstáculo. Por exemplo, se a face norte de um volume de controle consiste num elétrodo, sua contribuição será

$$
\int_{N} \partial_{y} \phi d S=\frac{\phi_{N}-\phi_{i, j}}{\left(\hat{Y}_{j+1}-\hat{Y}_{j}\right) / 2}\left(X_{i+1}-X_{i}\right)
$$

onde $\phi_{i, j+1}=\phi_{N}$ é a contribuição da voltagem imposta no norte. Aqui utiliza-se metade da distância pois considera-se que o valor é imposto no extremo do eletrodo. Para o caso onde a face norte consiste numa parede ou obstáculo não há fluxo nessa face, portanto $\int_{N} \partial_{y} \phi d S=0$. As condições de contorno influenciam os fluxos nas demais faces de maneira análoga. 
Uma vez que tem-se a solução do potencial elétrico no centro das células pode-se avaliar o campo elétrico nas componentes horizontais da velocidade, situadas no meio das faces leste e oeste, e nas componentes verticais, situadas no meio das faces norte e sul de cada volume de controle correspondente. Para proceder discretiza-se o gradiente do potencial elétrico de acordo com a equação (15).

Por exemplo, para calcular o campo elétrico na direção $x_{1}$ na face leste da célula $(i, j)$, faz-se

$$
E_{x_{1}}^{E}=-\nabla \Phi_{i, j}^{E}=-\frac{\phi_{i+1, j}-\phi_{i, j}}{\hat{X}_{i+1}-\hat{X}_{i}}
$$

Se a célula $(i+1, j)$ for eletrodo, tem-se

$$
E_{x_{1}}^{E}=-\frac{\phi_{E}-\phi_{i, j}}{\left(\hat{X}_{i+1}-\hat{X}_{i}\right) / 2},
$$

onde $\phi_{i+1, j}=\phi_{E}$ é a contribuição da voltagem imposta no leste. Aqui utiliza-se metade da distância pois considera-se que o valor é imposto no extremo do eletrodo. Para o caso onde a face leste consiste numa parede ou obstáculo o campo elétrico é nulo nessa face, $E_{x_{1}}^{E}=0$, de acordo com a condição de Neumann. Considerações similares são utilizadas para a face oeste. Para calcular o campo elétrico na direção $x_{2}$ nas faces norte e sul também procede-se de maneira análoga.

\subsection{Estudo de caso: soluções para os campos de velocidades}

Foram escolhidos alguns casos para ilustrar os campos de velocidade a partir dos quais serão determinadas as trajetórias possíveis para os pontos do domínio. A Figura 6 mostra o caso considerado.

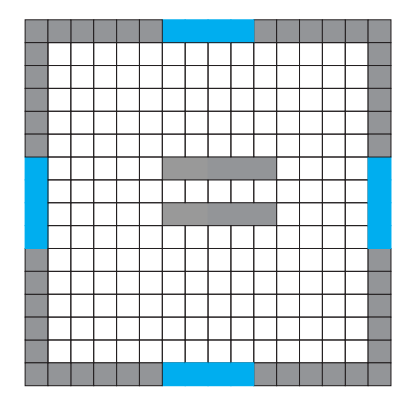

Figura 6: Configuração física considerada.

As Figuras 7 e 9 ilustram os campos de pressão e potencial resultantes, respectivamente, de diferentes combinações de pressões e voltagem impostas nos atuadores. Note que, quando tem-se 1 para pressão ou potencial no oeste, sempre ocorre saída de fluxo simétrica, e quando tem-se 1 no norte ou no sul a saída de fluxo ocorre mais intensamente para o atuador em frente e para o do leste, para o caso de quando é imposta pressão, e de maneira mais suave para todos os atuadores quando potenciais são considerados.

As Figuras 8 e 10 ilustram os campos de velocidade resultantes para as diferentes combinações de pressões e potenciais, respectivamente, impostas nos atuadores. 


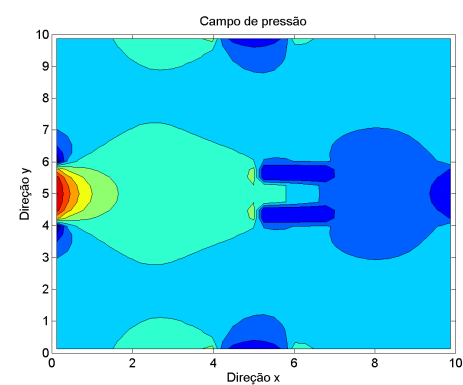

(a) $p_{N}=0, p_{S}=0, p_{W}=1$.

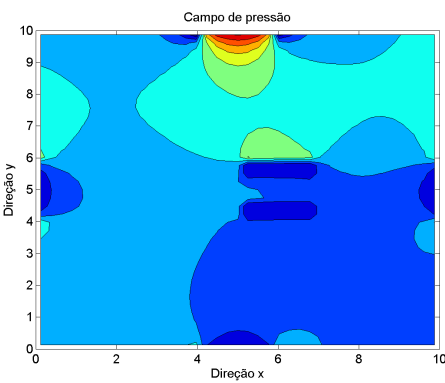

(b) $p_{N}=1, p_{S}=0, p_{W}=0$.

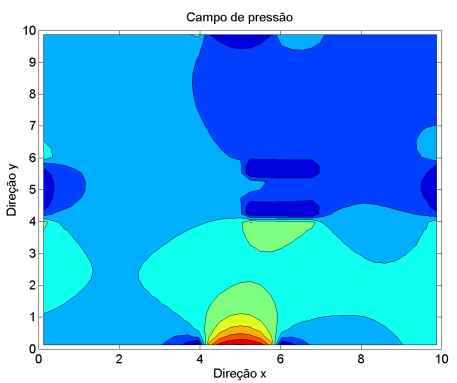

(c) $p_{N}=0, p_{S}=1, p_{W}=0$.

Figura 7: Campos de pressões para três diferentes combinações de pressão nos atuadores.

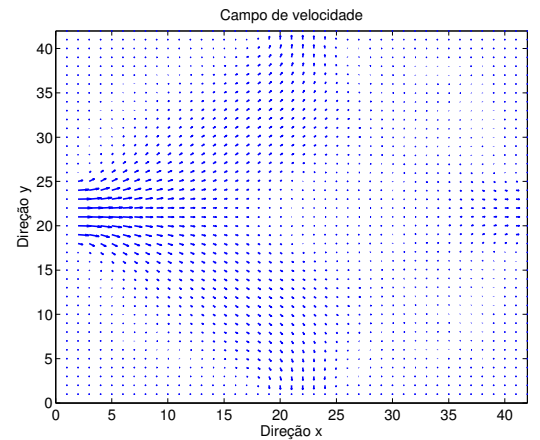

(a) $p_{N}=0, p_{S}=0, p_{W}=1$.

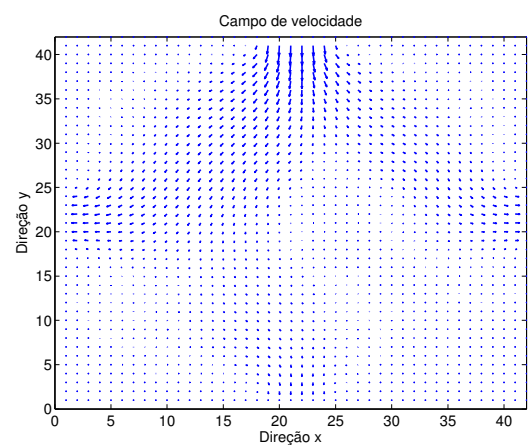

(b) $p_{N}=1, p_{S}=0, p_{W}=0$.

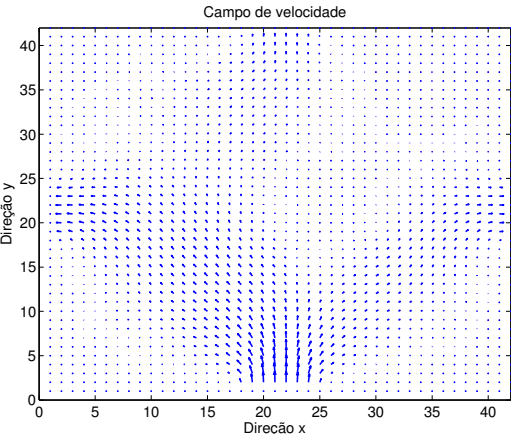

(c) $p_{N}=0, p_{S}=1, p_{W}=0$.

Figura 8: Campos de velocidades para as três diferentes combinações de pressão nos atuadores.

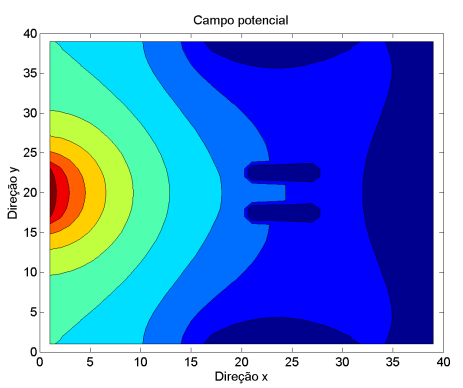

(a) $\phi_{N}=0, \phi_{S}=0, \phi_{W}=1$.

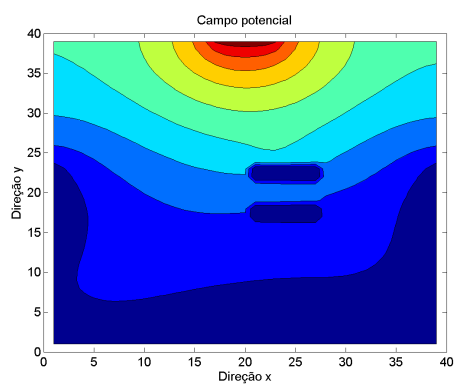

(b) $\phi_{N}=1, \phi_{S}=0, \phi_{W}=0$.

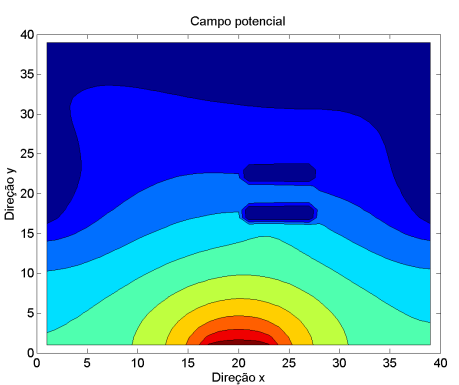

(c) $\phi_{N}=0, p h i_{S}=1, \phi_{W}=0$.

Figura 9: Campos potenciais para três diferentes combinações de potenciais nos atuadores.

No apêndice encontram-se outros casos ilustrados, onde é possível perceber como os obstáculos influenciam nos campos de velocidades.

\section{Resultados}

O tempo mínimo para o transporte de uma partícula foi determinado pela resolução da equação (4), que é uma simplificação para a proposição que trata numericamente a equação de HamiltonJacobi-Bellman (3). Para simplificação, os resultados apresentados consideram o máximo da 


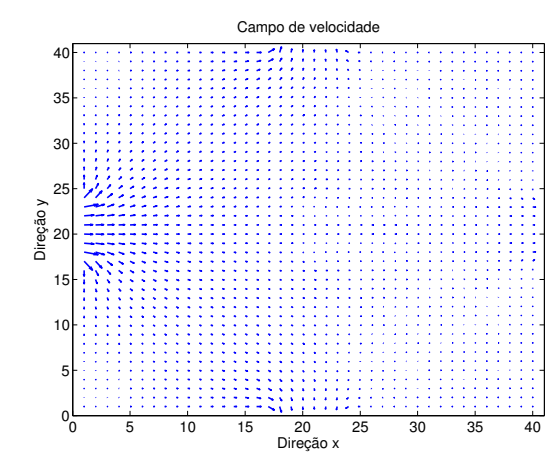

(a) $\phi_{N}=0, \phi_{S}=0, \phi_{W}=1$.

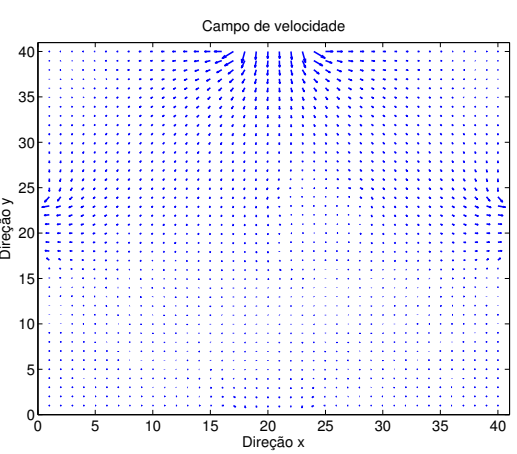

(b) $\phi_{N}=1, \phi_{S}=0, \phi_{W}=0$.

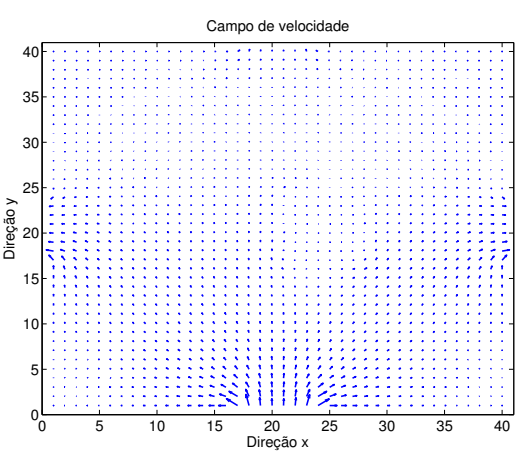

(c) $\phi_{N}=0, \phi_{S}=1, \phi_{W}=0$.

Figura 10: Campos de velocidades para as três diferentes combinações de potenciais nos atuadores.

velocidade, na equação (2), para 8 direções possíveis em cada ponto, partindo da direção leste e rotacionando-se de um ângulo de $\frac{\pi}{4}$. Com o tempo mínimo identificou-se a trajetória ótima para o deslocamento da partícula.

Considera-se o caso onde o ponto está próximo aos obstáculos, que são duas placas horizontais paralelas. Na Figura 11 está ilustrado um mapa contendo os tempos mínimos necessários para chegar de cada ponto do domínio ao ponto determinado como ponto objetivo. Observa-se que as regiões adjacentes ao contorno e aos obstáculos, decorrente das baixas velocidades do fluido, têm uma elevado tempo de trajetória, o que indica a baixa mobilidade de partículas situadas ou posicionadas nas referidas regiões. Para ambas as figuras foram utilizados atuadores do tipo voltagem, e portanto, um campo elétrico foi gerado. O limite mínimo de voltagem permitida considerado é -1 e o máximo é 1.

O modelo também é extremamente sensível às condições limitantes para os atuadores. Em todos os casos considerados foram utilizadas pressão ou voltagem mínimas de - 1 e máximas de 1, veja na Figura 12. Já na Figura 13 tem-se os resultados para as trajetórias quando as voltagem e pressão mínimas são alteradas para -0.1 mantendo-se as máximas em 1 . Percebe-se que para movimentar partículas da direita para a esquerda torna-se mais difícil. Devido a não simetria para os limites nos atuadores, o fluxo negativo, ou seja de volta, é mais demorado.

O modelo desenvolvido, embora seja uma primeira abordagem para o problema, mostrou que a ocorrência das trajetórias ótimas podem ser não intuitivas. Na Figura 14 consideram-se o ponto de chegada em azul e dois possíveis pontos de partida em vermelho. Para o ponto vermelho à esquerda o modelo propõe uma trajetória ótima reta. Quando avalia-se o tempo de percurso para uma reta ligando tal ponto ao ponto objetivo obtém-se o mesmo tempo proposto pela resolução de Hamilton-Jacobi. Isso confirma que o modelo proposto está bem definido. No caso do outro ponto vermelho, a trajetória ótima proposta pelo modelo não é reta. A mesma assume uma forma que não é intuitiva. Todavia o caminho proposto, apesar de ser mais longo do que o trajeto reto, resulta em um tempo menor do que quando percorre-se a trajetória reta. Esse deslocamento se dá devido à ação dos atuadores e a configuração do domínio. O resultado considerado é gerado para atuadores do tipo pressão com limite mínimo -1 e máximo 1 e as trajetórias discutidas estão em destaque. 


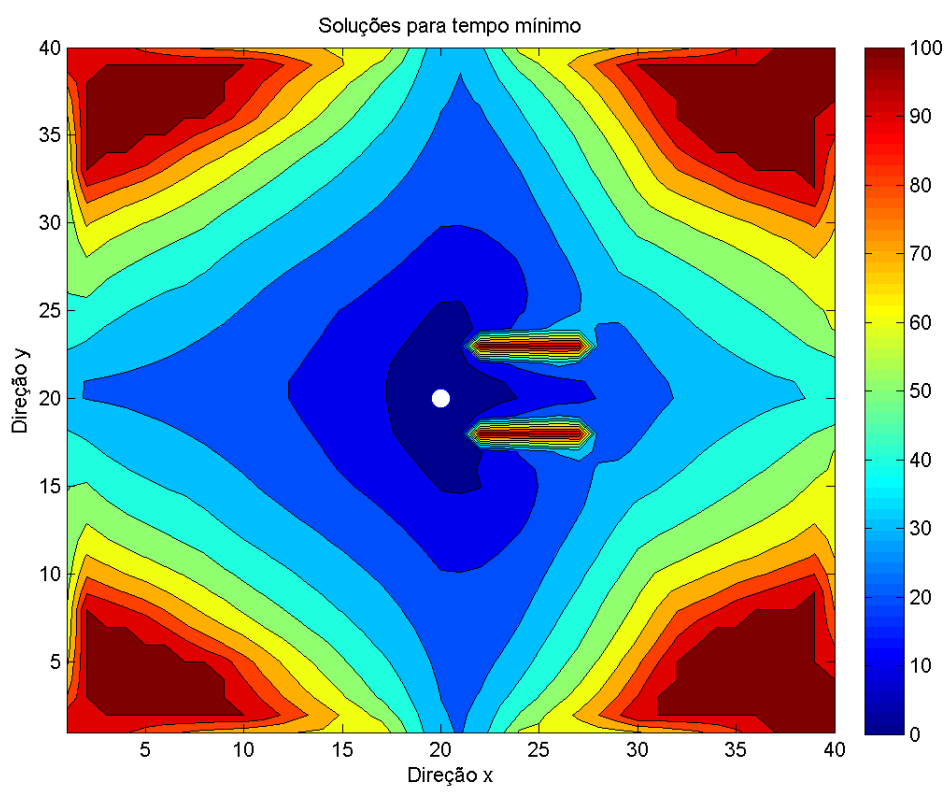

(a) Tempo mínimo.

Figura 11: Mapa com o tempo mínimo necessário para deslocar cada ponto do domínio até o ponto objetivo que está em branco, considerando-se a configuração física da Figura 6, atuadores do tipo potencial elétrico e uma malha com $40 \times 40$ células.

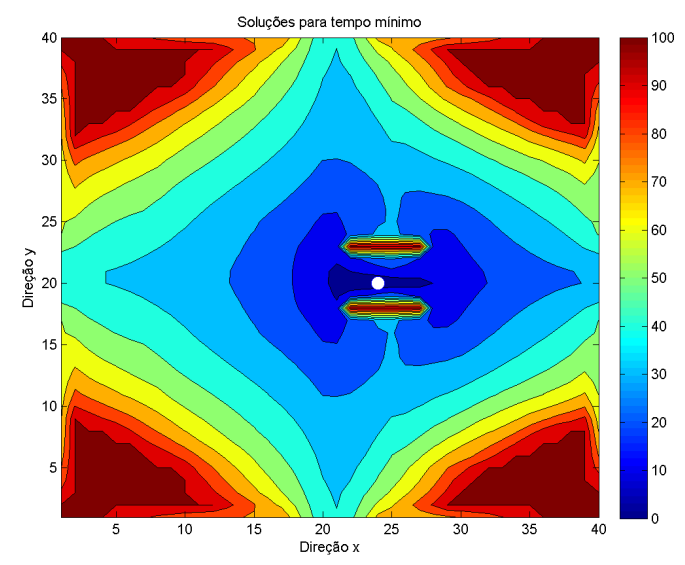

(a) Potencial elétrico.

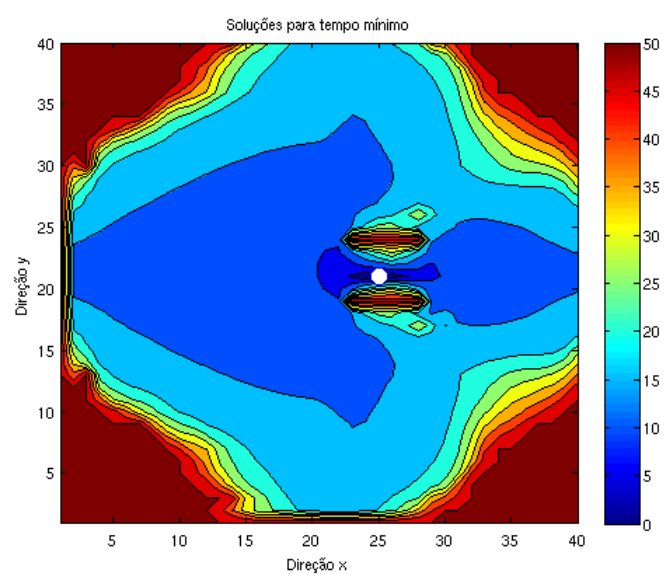

(b) Stokes.

Figura 12: Comparação de tempo mínimo.

\section{Conclusão}

Este trabalho é uma primeira abordagem para o problema de planejamento, no entanto, estabelece a base teórica da ferramenta para o estudo deste problema. O primeiro resultado obtido é a formulação matemática do tema em questão, que relaciona formalmente os problemas matemáticos envolvidos como a manipulação de trajetória, o problema de maximização das velocidades admissíveis, de tempo mínimo e de caminho ótimo. Apesar de utilizar uma geometria 


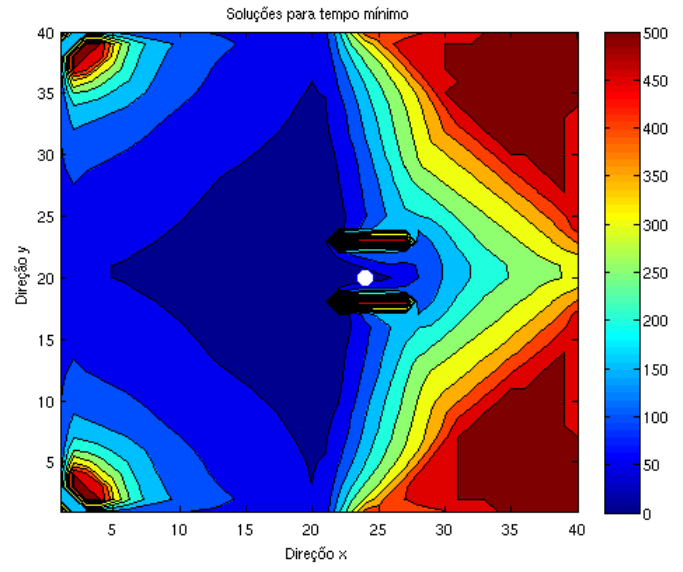

(a) Potencial elétrico.

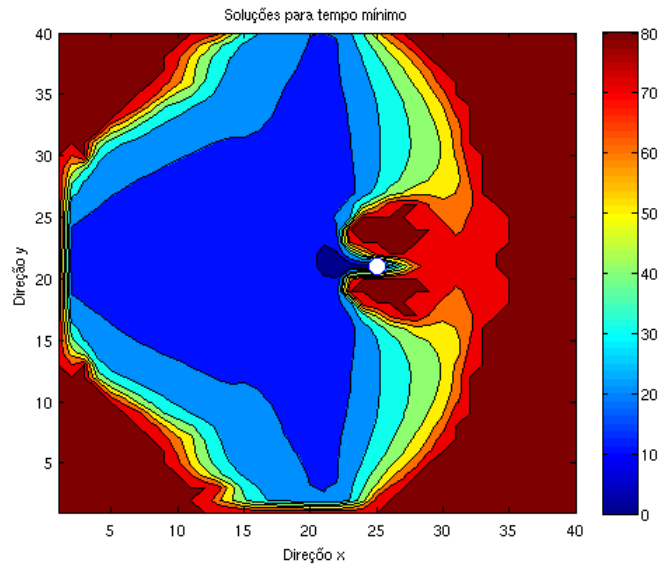

(b) Stokes.

Figura 13: Comparação de tempo mínimo mudando-se o limite mínimo nos atuadores para -0.1.

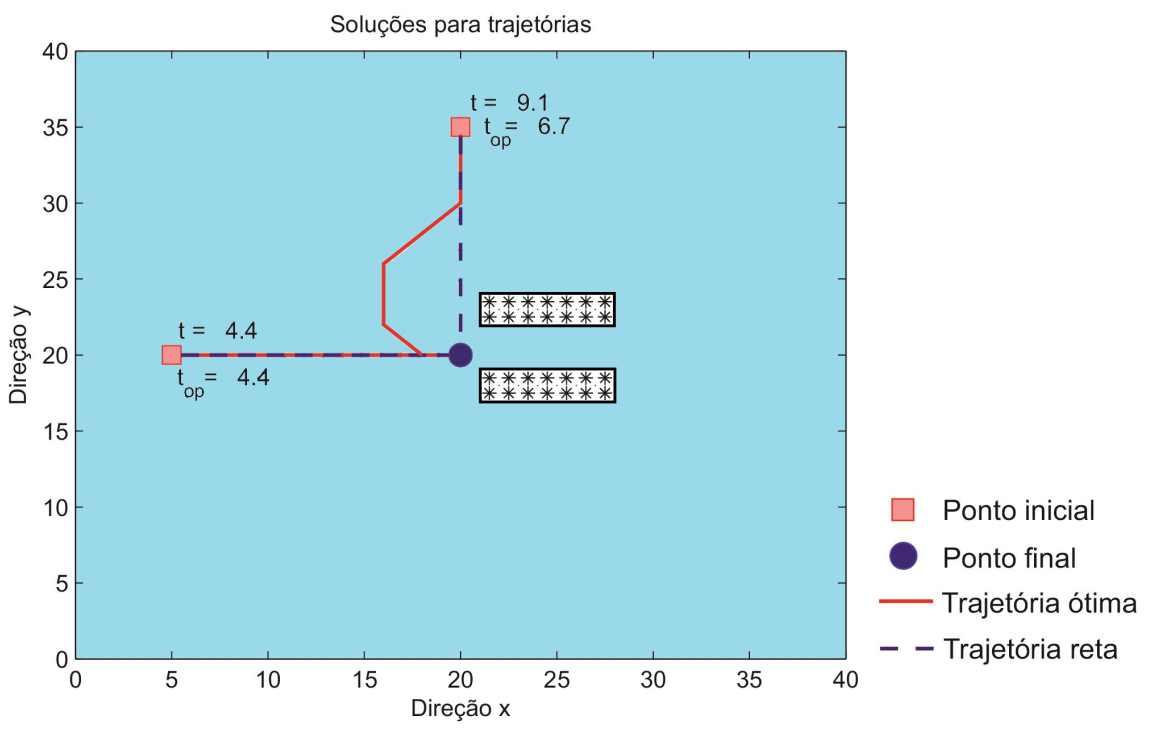

Figura 14: Trajetória de Hamilton-Jacobi-Bellman (ótima) comparada com a trajetória reta para atuadores do tipo pressão. No caso mostrado, seguir o caminho reto leva mais tempo $(t=9.1)$ em comparação com a trajetória ótima que é não intuitiva e mais longa $\left(t_{o p}=6.7\right)$.

física particular foi possível identificar como essa configuração interfere na trajetória de uma partícula. Para planejar a trajetória de partículas imersas em um fluido, considerou-se como trajetória ótima aquela que minimiza o tempo de deslocamento da partícula até o ponto alvo. Para isso foi necessário o cálculo e discretização do conjunto das velocidades máximas permitidas associadas à uma direção $\breve{d}$. O entendimento do conjunto das velocidades máximas foi fundamental para o desenvolvimento realizado. Inclusive o cálculo prévio do mesmo, que já considera as condições limitantes nos atuadores do sistema, permitiu o cálculo do tempo mínimo, que determina nesse trabalho a trajetória ótima, de acordo com o princípio de Bellman. Os resultados obtidos confirmam que esse tipo de assunto não é trivial, como mostra a ocorrência de trajetórias 
ótimas não intuitivas. Isso justifica a necessidade e importância desse tipo de abordagem matemática do estudo de planejamento de trajetórias. Este trabalho abre caminho para desenvolvimentos futuros para a manipulação de partículas em microfluídica.

\section{Agradecimentos} CNPq.

O presente trabalho foi realizado com o apoio financeiro das agências de fomento CAPES e

\section{Referências bibliográficas}

ARMANI, M. et al. Control of microfluidic systems: two examples, results, and challenges. International Journal of Robust and Nonlinear Control, v. 15, n. 16, p. 785 - 803, 2005.

BUSCAGLIA, G. C. Introduction to computational fluid dynamics. São Carlos: USP/ICMC, 2016. p. 148. Notas de Aula.

CHAUDHARY, S.; SHAPIRO, B. Arbitrary steering of multiple particles independently in an electro-osmotically driven microfluidic system. IEEE Transactions on Control Systems Technology, v. 14, n. 4, p. $669-680,2006$.

CRISTIANI E. Numerical methods for optimal control problems: part II: local single-pass methods for stationary HJ equations. Roma: IAC-CNR, 2013. p.21. Notas de Aula.

MEACCI, L. et al. Planejamento de trajetória para a manipulação de partículas em microfluídica. In: ENCONTRO REGIONAL DE MATEMÁTICA APLICADA E COMPUTACIONAL, 4.,2017, Bauru. Caderno de trabalhos completos e resumos. Bauru: Unesp, Faculdade de Ciências, 2017. p. 515 - 517.

TUVAL, I. et al. Control of particles in microelectrode devices. Physical Review Letters, v. 95, n.23, p. 1-4, 2005. 


\section{Apêndice: exemplos de outras geometrias possíveis}

Foram escolhidos alguns outros casos para ilustrar os campos de velocidade. A Figura 6 mostra os casos considerados.

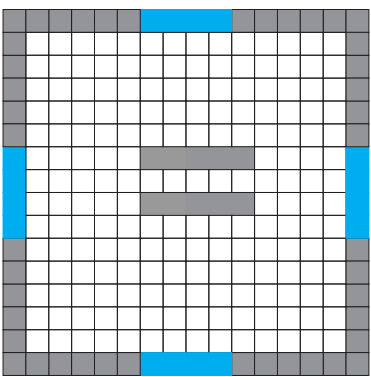

(a) Estudo de caso 1

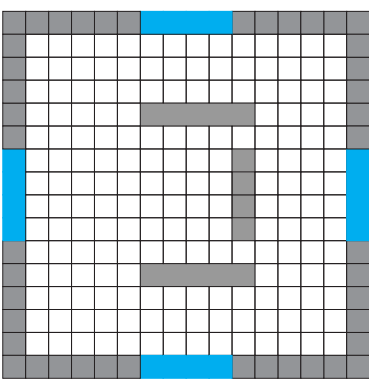

(b) Estudo de caso 2.

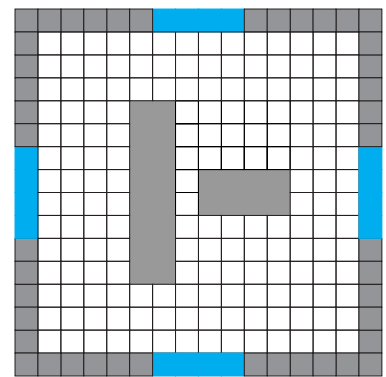

(c) Estudo de caso 3.

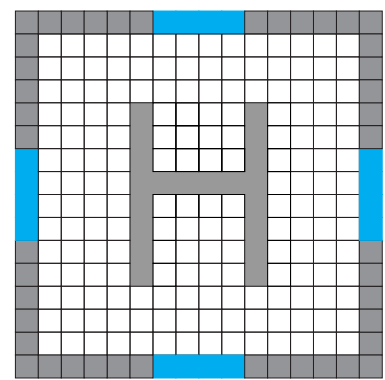

(d) Estudo de caso 4.

Figura 15: Diferentes tipos de obstáculos considerados.

\section{Estudo de caso 2}

As Figuras 16 e 18 ilustram os campos de pressão e potencial resultantes de diferentes combinações de pressões e potenciais impostas pelos atuadores. Note que, de maneira similar ao caso 1 , quando tem-se 1 para pressão ou potencial no oeste, sempre ocorre saída de fluxo simétrica, e quando tem-se 1 no norte ou ou sul a saída de fluxo ocorre mais intensamente para o atuador em frente, para o caso de quando é imposta pressão; e de maneira mais suave para todos os outros

atuadores quando potenciais elétricos são impostos.

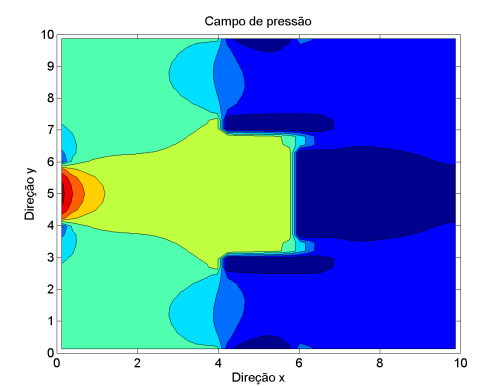

(a) $p_{N}=0, p_{S}=0, p_{W}=1$.

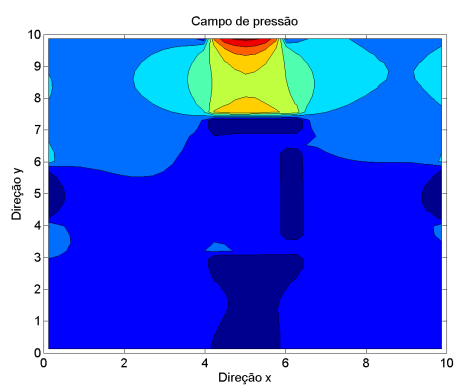

(b) $p_{N}=1, p_{S}=0, p_{W}=0$.

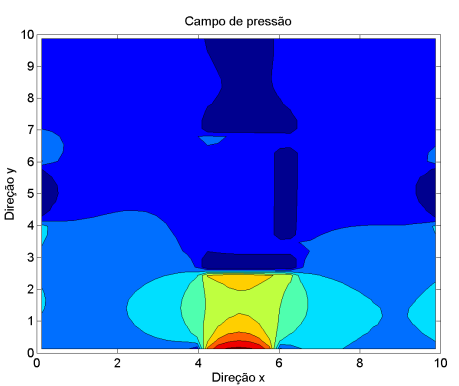

(c) $p_{N}=0, p_{S}=1, p_{W}=0$.

Figura 16: Campo de pressões para três diferentes combinações de condições de pressão nos atuadores.

As Figuras 17 e 19 ilustram os campos de velocidade resultantes para as diferentes combinações de pressões e potenciais impostos, respectivamente, nos atuadores. 


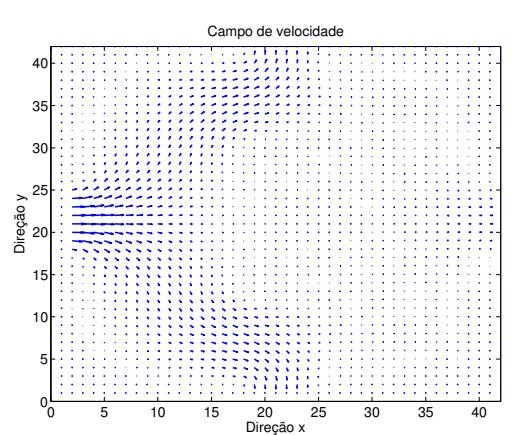

(a) $p_{N}=0, p_{S}=0, p_{W}=1$.

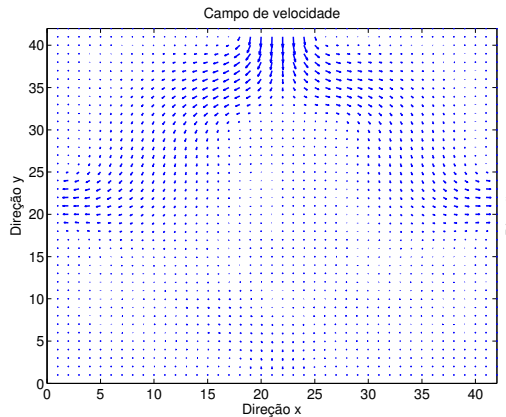

(b) $p_{N}=1, p_{S}=0, p_{W}=0$.

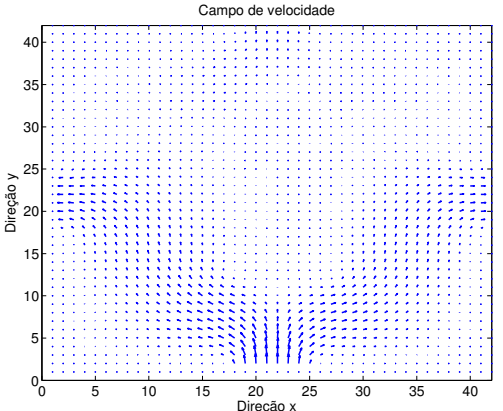

(c) $p_{N}=0, p_{S}=1, p_{W}=0$.

Figura 17: Campo de velocidades para as três diferentes combinações de condições de pressão nos atuadores.

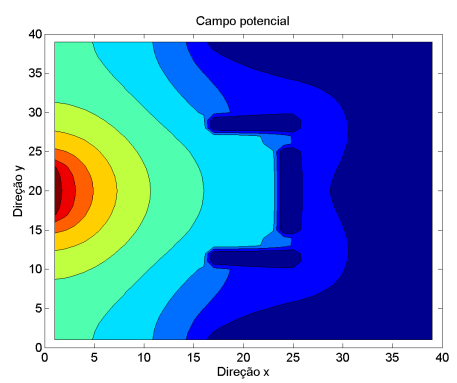

(a) $\phi_{N}=0, \phi_{S}=0, \phi_{W}=1$.

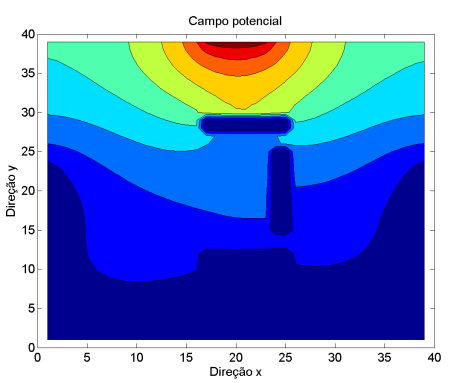

(b) $\phi_{N}=1, \phi_{S}=0, \phi_{W}=0$.

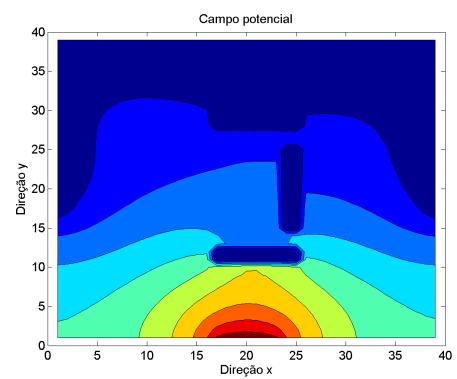

(c) $\phi_{N}=0, \phi_{S}=1, \phi_{W}=0$.

Figura 18: Campo de potenciais para três diferentes combinações de condições de potencial nos atuadores.

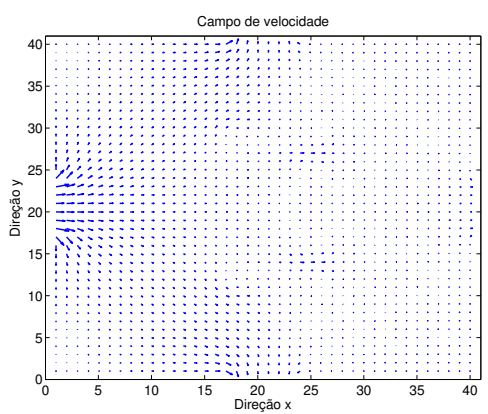

(a) $\phi_{N}=0, \phi_{S}=0, \phi_{W}=1$.

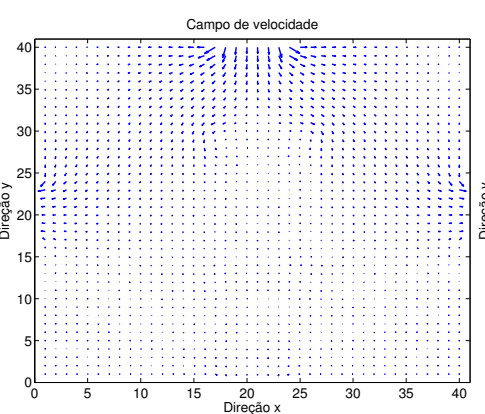

(b) $\phi_{N}=1, \phi_{S}=0, \phi_{W}=0$.

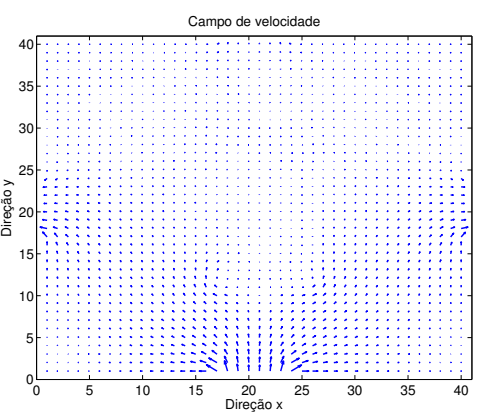

(c) $\phi_{N}=0, \phi_{S}=1, \phi_{W}=0$.

Figura 19: Campo de velocidades para as três diferentes combinações de condições de potenciais nos atuadores.

\section{Estudo de caso 3}

As Figuras 20 e 22 ilustram os campos de pressão e potencial resultantes de diferentes combinações de pressões e potenciais, respectivamente, impostas nos atuadores. Os resultados são similares ao caso tratado anteriormente, com pequenas variações que refletem a influência do tipo de obstáculo. As Figuras 21 e 23 ilustram os respectivos campos de velocidades. 


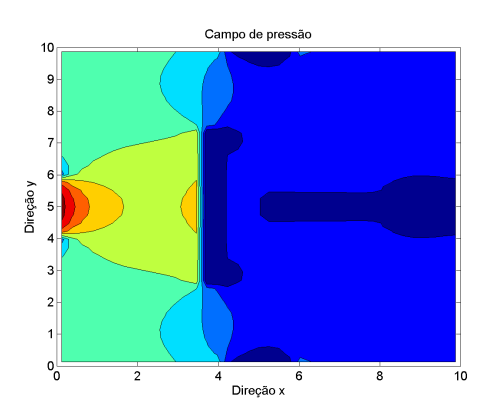

(a) $p_{N}=0, p_{S}=0, p_{W}=1$.

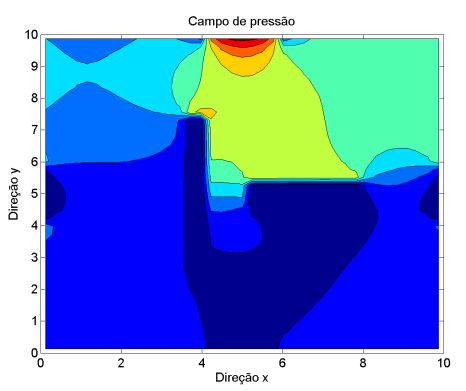

(b) $p_{N}=1, p_{S}=0, p_{W}=0$.

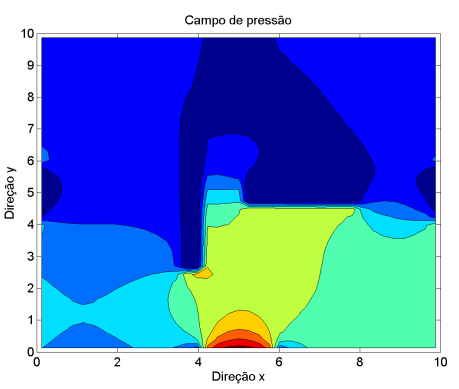

(c) $p_{N}=0, p_{S}=1, p_{W}=0$.

Figura 20: Campo de pressões para três diferentes combinações de condições de pressão.

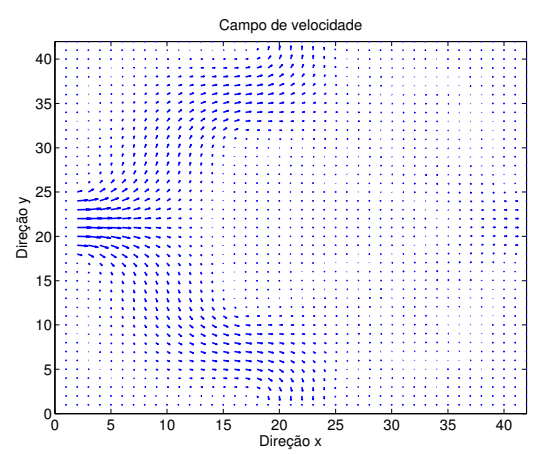

(a) $p_{N}=0, p_{S}=0, p_{W}=1$.

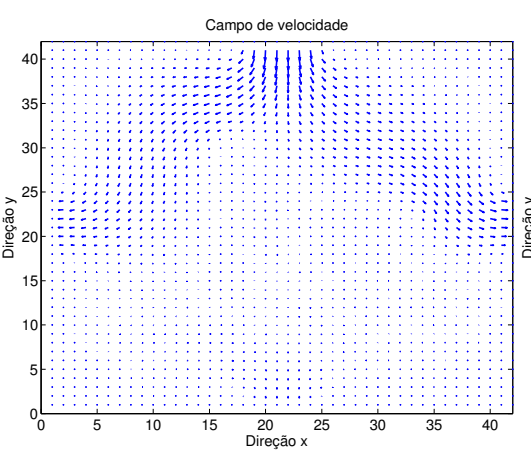

(b) $p_{N}=1, p_{S}=0, p_{W}=0$.

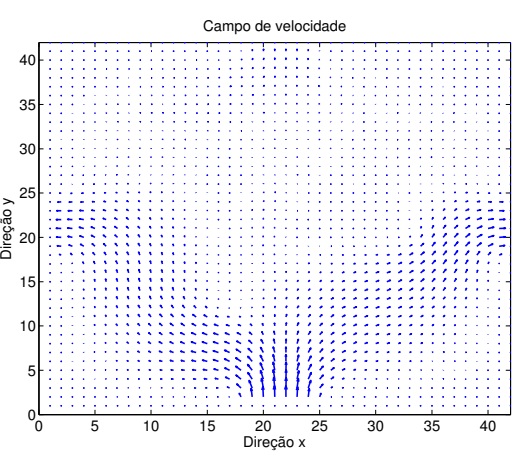

(c) $p_{N}=0, p_{S}=1, p_{W}=0$.

Figura 21: Campo de velocidades para as três diferentes combinações de condições de pressão.

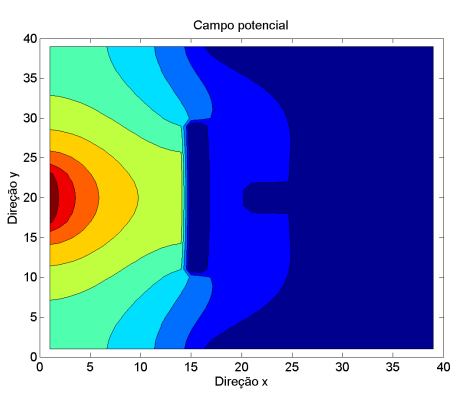

(a) $\phi_{N}=0, \phi_{S}=0, \phi_{W}=1$.

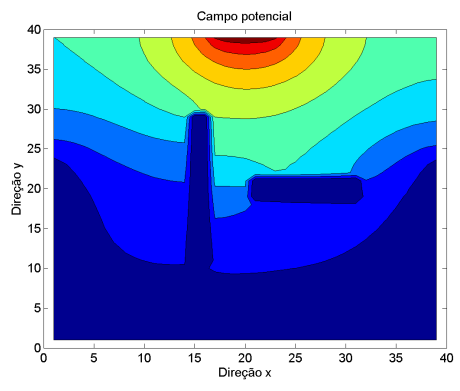

(b) $\phi_{N}=1, \phi_{S}=0, \phi_{W}=0$.

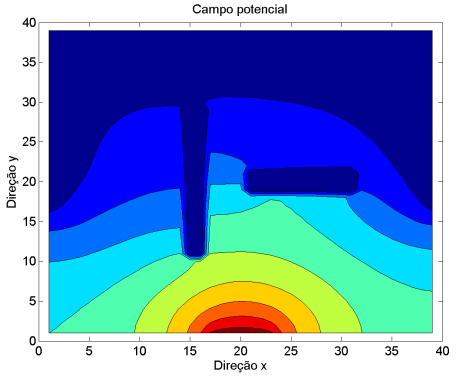

(c) $\phi_{N}=0, \phi_{S}=1, \phi_{W}=0$.

Figura 22: Campo de pressões para três diferentes combinações de condições de pressão.

\section{Estudo de caso 4}

As Figuras 24 e 26 ilustram os campos de pressão e potencial, onde observa-se resultados similares aos anteriores e percebe-se a influência do obstáculo no campo de pressão. As figuras 25 e 27 ilustram os respectivos campos de velocidade. 


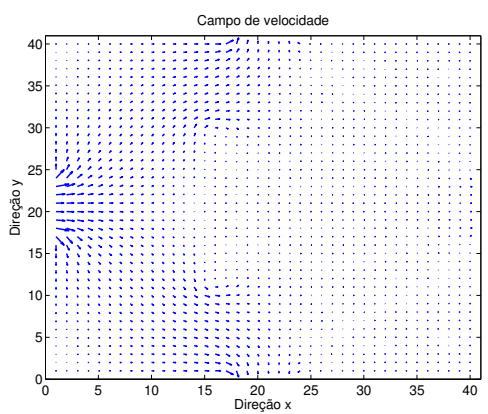

(a) $\phi_{N}=0, \phi_{S}=0, \phi_{W}=1$.

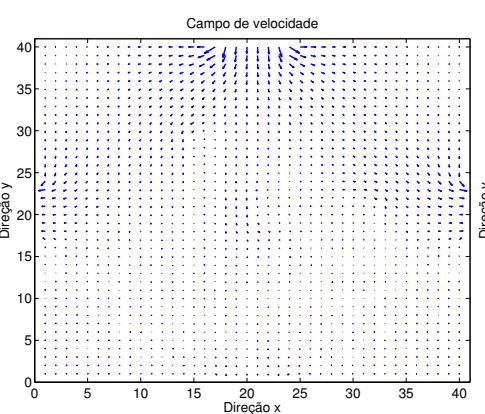

(b) $\phi_{N}=1, \phi_{S}=0, \phi_{W}=0$.

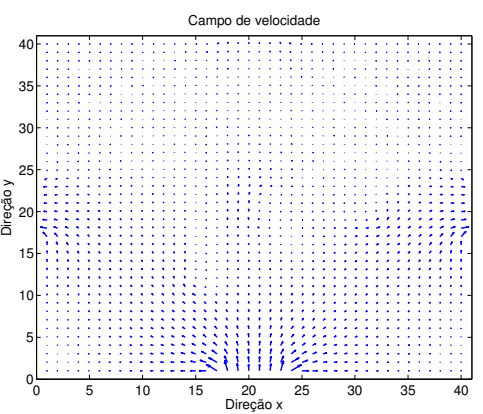

(c) $\phi_{N}=0, \phi_{S}=1, \phi_{W}=0$.

Figura 23: Campo de velocidades para as três diferentes combinações de condições de poteciais.

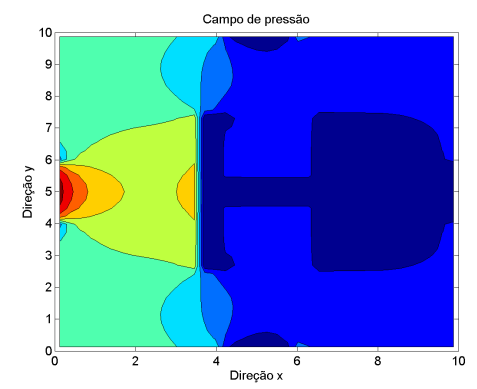

(a) $p_{N}=0, p_{S}=0, p_{W}=1$.

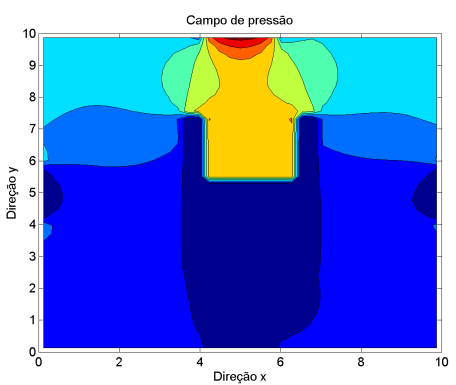

(b) $p_{N}=1, p_{S}=0, p_{W}=0$.

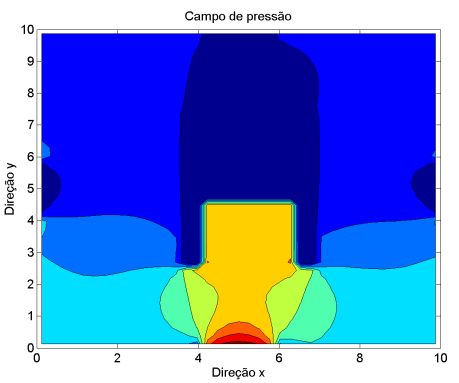

(c) $p_{N}=0, p_{S}=1, p_{W}=0$.

Figura 24: Campo de pressões para três diferentes combinações de condições de pressão.

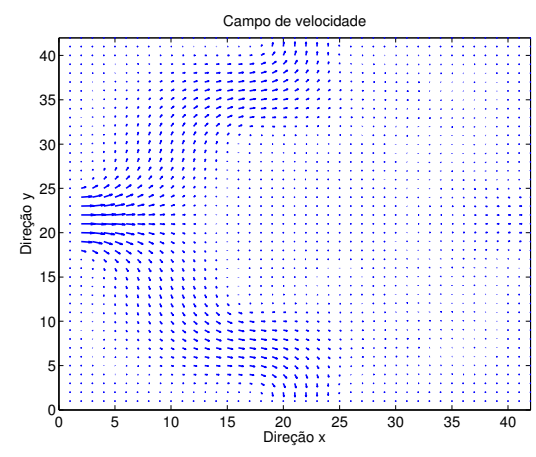

(a) $p_{N}=0, p_{S}=0, p_{W}=1$.

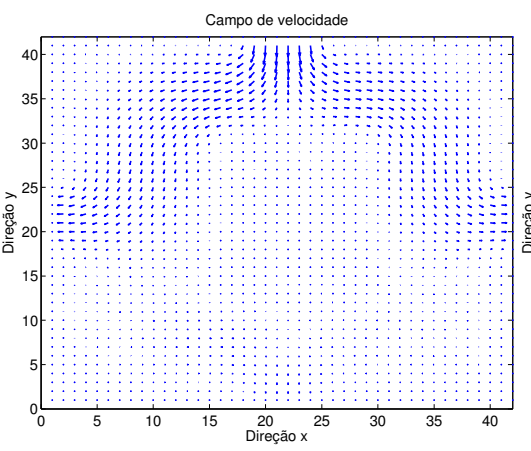

(b) $p_{N}=1, p_{S}=0, p_{W}=0$.

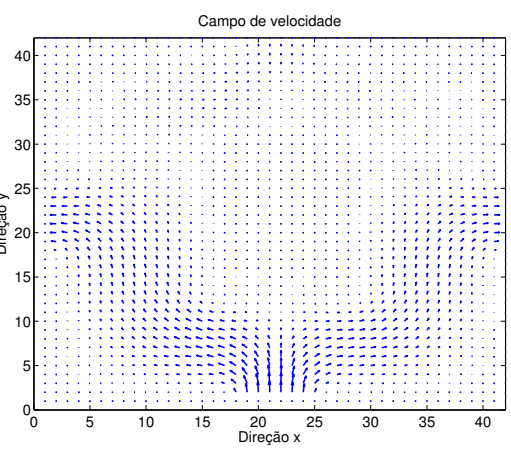

(c) $p_{N}=0, p_{S}=1, p_{W}=0$.

Figura 25: Campo de velocidades para as três diferentes combinações de condições de pressão. 


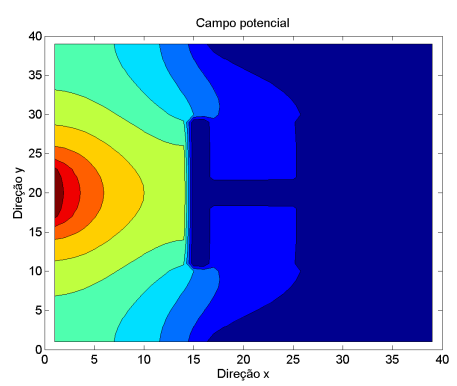

(a) $\phi_{N}=0, \phi_{S}=0, \phi_{W}=1$.

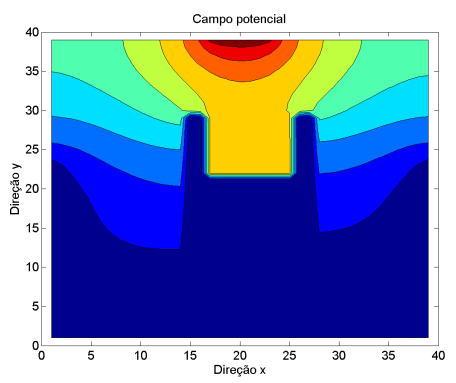

(b) $\phi_{N}=1, \phi_{S}=0, \phi_{W}=0$.

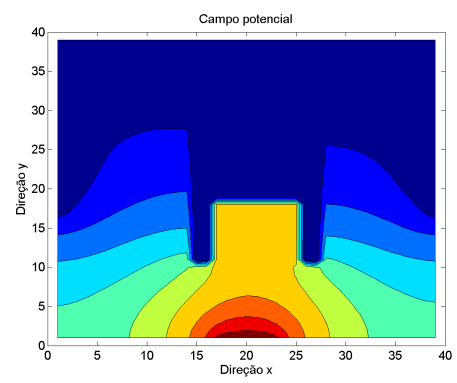

(c) $\phi_{N}=0, \phi_{S}=1, \phi_{W}=0$.

Figura 26: Campo potencial para três diferentes combinações de condições de tensões.

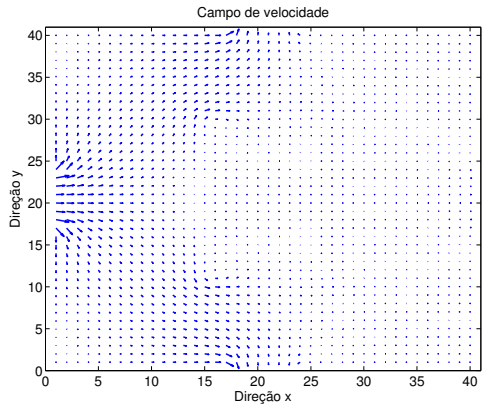

(a) $\phi_{N}=0, \phi_{S}=0, \phi_{W}=1$.

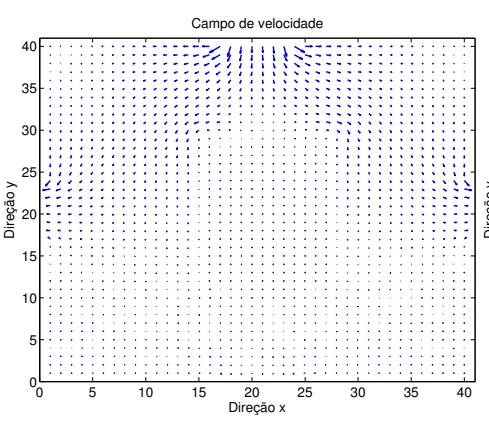

(b) $\phi_{N}=1, \phi_{S}=0, \phi_{W}=0$.

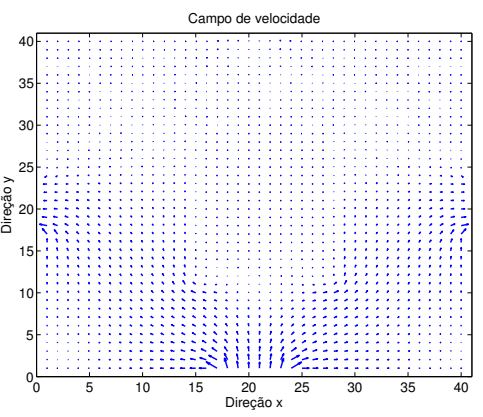

(c) $\phi_{N}=0, \phi_{S}=1, \phi_{W}=0$.

Figura 27: Campo de velocidades para as três diferentes combinações de condições de tensão.

Artigo recebido em jun. 2017 e aceito em ago. 2017. 